\title{
Umbilical Venous Catheter Complication Presenting as Chylous Ascites in a Newborn: Intraperitoneal Extravasa- tion of Total Parenteral Nutrition Infusate
}

Hye Mi Lee, Hyun Jung Sung, and Hyun-Seung Lee

Department of Pediatrics, College of Medicine, The Catholic University of Korea, Seoul, Korea

\section{ABSTRACT}

Umbilical venous catheterization (UVC) is a common practice in intensive neonatal care. However, a malpositioned UVC and its prolonged use may lead to various problems, including mechanical, infectious, and thrombotic complications in various organs such as the liver, lungs, and heart. Congenital chylous ascites is characterized by abnormally high levels of triglycerides in the peritoneal fluid of newborns, which originate from refluxed lymph within the abdominal cavity. Herein, we report a case of an UVC complication presenting as chyloperitoneum simulating congenital chylous ascites in a preterm neonate that resulted from total parenteral nutrition (TPN) extravasation from a malpositioned UVC. Biochemical analysis of intraperitoneal chylous fluid and TPN infusate could help confirm the origin of chyloperitoneum. This case suggests that TPN extravasation from UVC should be considered when chyloperitoneum develops in newborns with an indwelling catheter. UVC positions must also be carefully monitored at regular intervals to recognize associated complications early, particularly in cases with an inevitably malpositioned catheter related to the anatomy of the vessel course.

Key Words: Umbilical venous catheter, Chylous ascites, Total parenteral nutrition, Newborn

\section{INTRODUCTION}

Umbilical venous catheterization (UVC) has been widely used for central venous access to provide nutritional support in the intensive care of prematurely born infants intolerant to enteral supplementation. However, it may cause various complications due to inadequate positioning of $\mathrm{UVCs}^{1,2)}$. UVC malpositioning is related to the catheter tip, which is freely movable under physiological conditions such as lung expansion, diaphragmatic descent, and blood streaming force, or a failure in advancement of UVCs to an appropriate destination because of difficulty in negotiating certain anatomical sites ${ }^{1)}$. UVC malpositioning, along with prolonged dwelling of a UVC, may lead to mechanical, infectious,
Received: 3 September 2018

Revised: 20 October 2018

Accepted: 24 October 2018

Correspondence to: Hyun-Seung Lee Department of Pediatrics, Uijeongbu St. Mary's Hospital, College of Medicine, The Catholic University of Korea, 271 Cheonbo-ro, Uijeongbu 11765 , Korea

Tel: +82-31-820-3590

Fax: +82-31-821-3108

E-mail: iamlidia@catholic.ac.kr

Copyright(c)

By Korean Society of Neonatology.

All right reserved.

This is an Open-Access article distributed under the terms of the Creative Commons Attribution Non-Commercial License (http://creativecommons.org/licenses/ by-nc/4.0), which permits unrestricted non-commercial use, distribution, and reproduction in any medium, provided the original work is properly cited. 
and thrombotic complications of different organs such as the liver, lungs, and heart within or at 2 weeks after UVC insertion ${ }^{1,-5}$.

Ascites is an intraperitoneal collection of fluids (transudate, exudate, or chylous effusion) due to various causes, including vascular stream resistance due to portal hypertension, infection, inflammation, malignancy, and lymphatic system dysfunction ${ }^{6,7)}$. Congenital fetal or neonatal chylous ascites is characterized by the abnormally high levels of triglycerides in the peritoneal fluid, which originates from lymph (containing lymphoid cells, protein, and chylomicrons) that is prevented from circulating toward the venous system ${ }^{8}$. This is due to a wide range of congenital conditions that lead to lymphatic vessel impairment or obstruction within the abdominal cavity ${ }^{7,8)}$. The refluxed chyle derives from a dietary source with long-chain triglycerides after birth $^{8)}$

Herein, we report a case of a UVC complication that occurred in a preterm neonate and presented as an intraperitoneal chylous fluid collection simulating congenital chylous ascites and resulted from total parenteral nutrition (TPN) extravasation due to improper UVC placement.

\section{CASE REPORT}

A Korean male infant with a birth weight of 2,960 g was born at $35^{+2}$ weeks of gestation via repeat cesarean section delivery. His immediate postnatal course was unremarkable except for

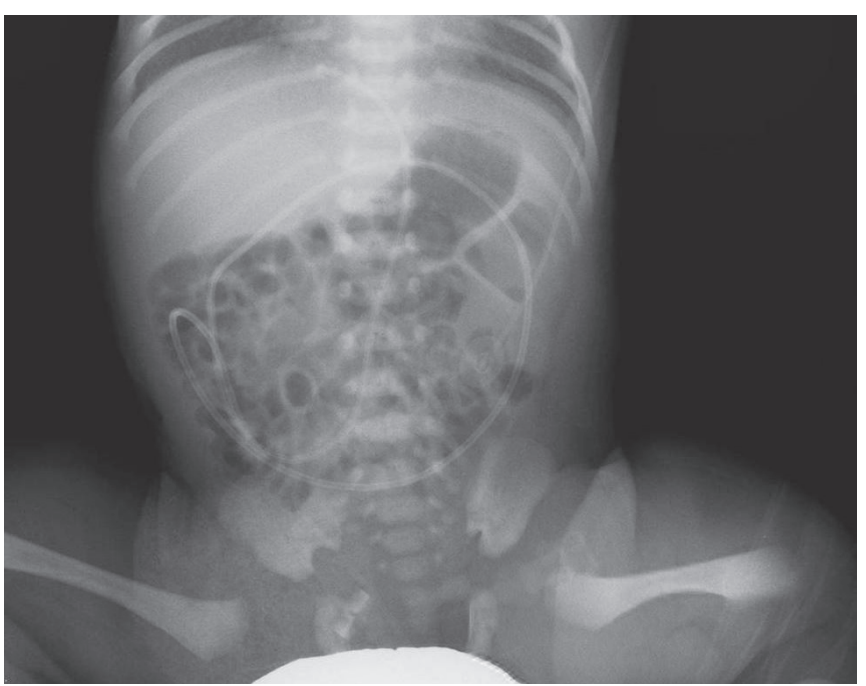

Figure 1. Abdominal radiography image obtained immediately after umbilical venous catheter insertion, showing the catheter tip within the liver shadow below the right diaphragm, indicating that the tip was likely located inside the right hepatic vein. mild respiratory distress syndrome, which responded well to conservative care. Gavage feeding was initiated but was not tolerated by the infant. Thus, an UVC was inserted for TPN on day 1 of life. The UVC insertion length was $10 \mathrm{~cm}$, which was calculated with Shukla's formula ${ }^{9}$, using birth weight in kilo. grams. Initial anteroposterior thoracoabdominal radiography revealed that the UVC tip was within the liver shadow below the right diaphragm (Figure 1), indicating that it might have been placed inside the right hepatic vein. Therefore, the catheter tip was withdrawn to $8 \mathrm{~cm}$, which was lower than the acceptable position, to avoid possible hepatic complications. On day 2 of life, the UVC tip was pulled to $6 \mathrm{~cm}$ because the catheter tip was suspected to be located within the left hepatic vein. Gavage feeds were still intolerable. A total of $10 \mathrm{ml}$ of standard preterm formula milk, Babywell Preemie (Maeil Dairies, Seoul, Korea), was administered for the first 2 days. At 13 hours after the final repositioning of the catheter tip, the infant developed abdominal distension and signs of poor perfusion. Thoracoabdominal radiography revealed a distended abdomen, with the UVC tip that was thought to be positioned within the section of the umbilical vein, traversing superficially, parallel to the cutaneous surface in lateral view (Figure 2). Respiratory distress occurred. His abdominal girth was markedly increased from 32 up to 40 $\mathrm{cm}$. He was afebrile. Abdominal ultrasonography revealed a large amount of ascites without other abnormalities, including hepatic injuries. UVC was removed and all feeds were discontinued.

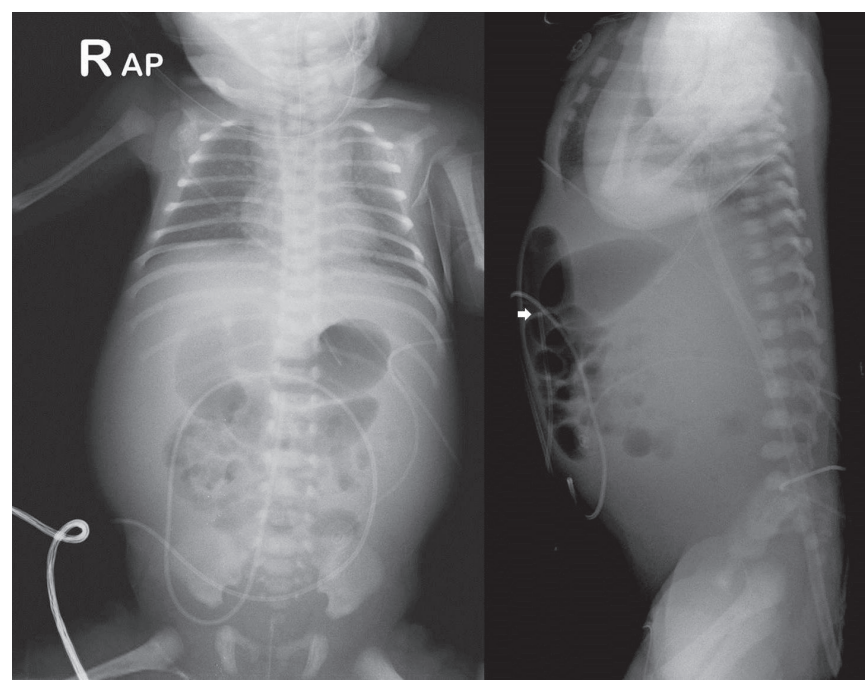

Figure 2. Anteroposterior radiography image of the abdomen depicting abdominal distension. The catheter tip (white arrow) was likely located at the portion of the umbilical vein, which was traversing superficially parallel to the cutaneous surface on lateral view. 
Computed tomography of the abdomen revealed consistent ultrasonography findings and pulmonary edema (Figure 3). Diagnostic and therapeutic paracentesis was performed. A total of $31 \mathrm{~mL}$ of milky-white chylous ascites was obtained (Figure 4), and $10 \mathrm{~mL}$ of chylous effusion was drained additionally. Initially, congenital chylous ascites and UVC complications were considered as differential diagnoses. Peritonitis was ruled out because no signs of infection were observed and his C-reactive protein level $(0.14 \mathrm{mg} / \mathrm{dL})$ was within the normal range. The infant was given ventilatory and inotropic supports and prophylactic antibiotics (cefotaxime $150 \mathrm{mg} / \mathrm{kg} /$ day) for 5 days. His abdominal

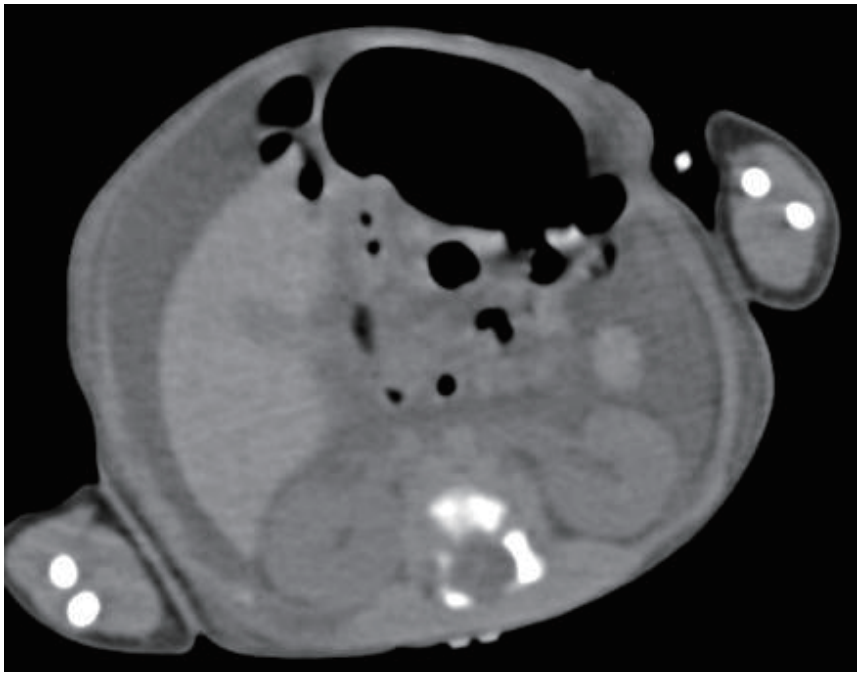

Figure 3. Computed tomography scan of the abdomen demonstrating a large amount of ascites.

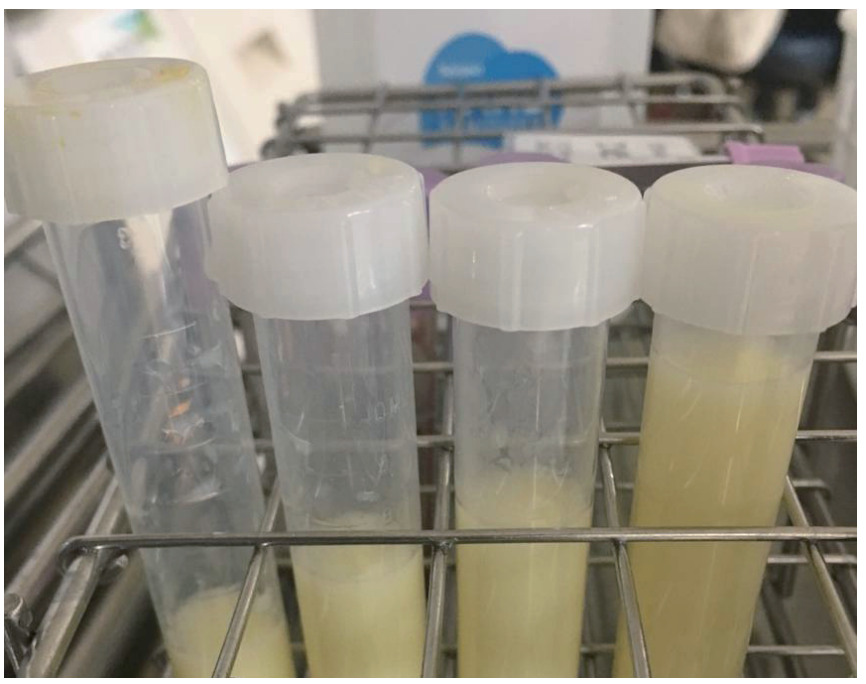

Figure 4. Abdominal paracentesis yielding $31 \mathrm{~mL}$ of milky-white chylous intraperitoneal fluid, which was extravasated with a total parenteral nutrition solution. girth was restored to $31 \mathrm{~cm}$ within 2 days after abdominal paracentesis. The hydroperitoneum or chyloperitoneum did not recur. Biochemical analysis of the obtained chylous intraperitoneal fluid revealed that the ascitic fluid originated from the hyperosmolar and acidic TPN solution, in which the lipid source was from a SMOFlipid 20\% injection (Fresenius Kabi Austria $\mathrm{GmbH}$, Graz, Austria) containing 30\% medium-chain triglycerides, and not from the lymph, transudates, or exudates (Table 1). Cytology tests for malignancy, acid-fast bacilli, Gram stains, and bacterial and fungal cultures were all negative. The infant was diagnosed as having intraperitoneal extravasation of TPN infusate from UVC. Enteral feeding was reintroduced on day 9 of life, and full enteral feeds were achieved on day 16 of life. The infant was discharged on day 21 of life.

\section{DISCUSSION}

The position of the inserted UVC is believed to be adequate when the catheter tip is located either at the inferior vena cava (IVC)-right atrial junction or in the intrathoracic portion of the IVC, which is approximately at the level of the diaphragm on abdominal radiography ${ }^{1)}$. Mutlu et al. ${ }^{2)}$ suggested that a catheter tip positioned between the $\mathrm{T} 9$ and $\mathrm{T} 10$ vertebrae is in the correct position. The acceptable and safe placement of an UVC is ascer-

Table 1. Analysis Results of Intraperitoneal Ascitic Fluid and TPN Infusates and Diagnostic Criteria for Chyloperitoneum from Refluxed Lymph

\begin{tabular}{|c|c|c|c|}
\hline Variable & $\begin{array}{l}\text { Chylous } \\
\text { ascites } \\
\text { analysis } \\
\text { results }\end{array}$ & $\begin{array}{c}\text { TPN } \\
\text { formula } \\
\text { analysis } \\
\text { results }\end{array}$ & $\begin{array}{c}\text { Criteria for } \\
\text { diagnosing } \\
\text { chylous ascites } \\
\text { from refluxed } \\
\text { lymph }\end{array}$ \\
\hline Triglyceride $(\mathrm{mg} / \mathrm{dL})$ & 869 & 984 & $>200$ \\
\hline Glucose (mg/dL) & 329 & 10,500 & \\
\hline Protein (g/dL) & 0.2 & 1.4 & $>2.5$ \\
\hline $\operatorname{WBC}\left(/ \mathrm{mm}^{3}\right)$ & $\begin{array}{c}145 \\
\text { (with } 79 \% \text { of } \\
\text { neutrophils) }\end{array}$ & & $\begin{array}{c}>1,000 \\
\text { (with lymphocyte } \\
\text { predominance) }\end{array}$ \\
\hline $\operatorname{RBC}\left(/ \mathrm{mm}^{3}\right)$ & 1,450 & & \\
\hline Color & Milky-yellow & Milky-yellow & Milk-like \\
\hline LDH (IU/L) & 93 & & $>110$ \\
\hline $\mathrm{pH}$ & 6.8 & 6.2 & \\
\hline Specific gravity & 1.013 & 1.080 & \\
\hline
\end{tabular}

Abbreviations: TPN, total parenteral nutrition; WBC, white blood cell; $\mathrm{RBC}$, red blood cell; $\mathrm{LDH}$, lactate dehydrogenase. 
tained largely by plain anteroposterior thoracoabdominal radiography immediately after UVC insertion. However, a risk of malpositioning still exists due to migration of the catheter tip, which may cause life-threatening complications ${ }^{1)}$. The investigation of choice to confirm the placement of UVC tips has been proposed to be ultrasonography, which is superior to plain radiography ${ }^{10,11)}$. The insertion length of the UVC has been determined in accordance with birth weight (Shukla's formula) and shoulder-to-umbilicus distance ${ }^{9,12)}$. However, it may lead to incorrect UVC placement, particularly in prematurely born and low-birth-weight infants ${ }^{1)}$.

To date, reported rates of UVC malpositioning range from $20 \%$ to $37 \%^{2,13)}$. Various complications, including hepatic, cardiac, and pulmonary lesions, have been found to be associated with UVC malpositioning depending on the location of the UVC tip. Hepatic problems (hematoma ${ }^{1,2,14)}$, abscess ${ }^{2)}$, laceration $^{15)}$, and transient portal venous gas ${ }^{2)}$ ) are frequently reported. They are related to low positioning of the UVC tip within the liver, inferior to the IVC. Cardiac (arrhythmia ${ }^{4)}$, left atrial thrombus ${ }^{1)}$, and pericardial effusion causing cardiac tamponade ${ }^{1)}$ ) and pulmonary problems (edema, hemorrhage, infarction, and hydrothorax) ${ }^{3)}$ have risks for more severe outcomes. They are related to high positioning of the UVC tip inside the heart or lungs, superior to the IVC. Other complications associated with UVC insertion include intraperitoneal TPN extravasation presenting as chylous ascites ${ }^{16-18)}$ as in our case; central line-related bloodstream infection, which occurs in up to $29 \%$ of central venous catheters placed ${ }^{19)}$, largely due to retained catheters and by coagulase-negative staphylo$\operatorname{cocci}^{4}$; and the presence of catheter remnant in the umbilicus after catheter removal ${ }^{2}$. In a 6-year retrospective observational study of 974 newborns, Mutlu et al. ${ }^{2)}$ reported that the prevalence of UVC-related complications was $20.3 \%$ (198 infants), of which malpositioning of the UVC tip (189 infants) is the most common as follows: low placement at $54 \%$ (102 patients), high placement at $32 \%$ (60 patients), and abnormal placement in the hepatic (26 patients) and splenic veins (one patient) at $14 \%$.

Chylous intraperitoneal effusion in newborns is generally suggestive of a diagnosis of congenital chylous ascites. However, as shown in our case, chyloperitoneum associated with UVC complications should be considered as a differential diagnosis. Biochemical analysis can be used to discriminate whether the cumulative fluid originates from refluxed lymph or extravasated TPN infusate. In our case, the analysis confirmed that the yielded chylous ascites was derived from the TPN solution (Table 1). In addition, the following were used to rule out congenital chylous ascites: (1) in TPN, unlike enteral feeds, the bowel was bypassed, and parenteral nutritional support was given with a lipid source from a SMOFlipid $20 \%$ injection containing 30\% medium-chain triglycerides, which did not require transport via the lymphatic system and contributed to chyle flow; (2) absence of ascites on fetal ultrasonography and lack of signs of ascites prior to the occurrence of abdominal distension after birth. Congenital chylous ascites is known to manifest as fetal and postnatal ascites of transudates ${ }^{7,8)}$.

The mechanism of chyloperitoneum by TPN extravasation from a malpositioned UVC can be postulated to occur in the following two ways: (1) The UVC fails to enter the IVC because of difficulty in negotiating the anatomical site, which may result in the UVC tip moving into one of the portal vein branches or hepatic veins within the liver. Catheter tip movement in the vessel along with an infused and flushed hyperosmolar or acidic TPN solution can lead to vascular and hepatic erosion and damage with extravasation of the TPN fluid, eventually forming a subcapsular accumulation of TPN infusate and/or blood via the path through the liver parenchyma because of least resistance. Chyloperitoneum can occur when the subcapsular fluid collection ruptures ${ }^{1,2,20)}$; (2) A much lower positioned UVC tip such as inside the umbilical vein may cause direct TPN fluid leakage via micropunctures of the vascular wall into the peritoneal cavity. In severe cases, the catheter tip may penetrate the vascular wall. In our case, the latter is more plausible despite the absence of sonographic confirmation for the UVC tip position. This may explain the prompt occurrence of chyloperitoneum at 21 hours after final repositioning of the catheter tip, unlike other reports of hepatic complications presenting at 3 to 9 days after UVC insertion $^{1,2,14)}$. When abdominal distension developed, no intrahepatic lesion was found on ultrasonography, while plain radiography revealed the catheter tip likely located at the level of the umbilical vein.

In this patient, lower placement of the UVC was related to the anatomical shape of the path of the umbilical vein-IVC, which made it difficult for the UVC tip to advance to the IVC. The umbilical vein was observed traversing superficially parallel to the skin surface on lateral radiography (Figure 2), forming a circuitous path with a lower angle compared with the typical straight course between the umbilical vein and the IVC.

Some cases of intraperitoneal TPN extravasation from an UVC have been reported in the English literature ${ }^{16-18)}$. Coley et al. ${ }^{18)}$ 
reported eight neonates with TPN ascites with low placement (intrahepatic location) of UVCs. They suggested that infused hypertonic TPN via UVC can produce local tissue necrosis and may disrupt the liver capsule, resulting in free intraperitoneal spillage of TPN ${ }^{18}$. No reports have described biochemical comparisons of the yielded ascitic fluid and TPN infusates ${ }^{16-18)}$. The biochemical analyses that was carried out in our study was useful in proving the origin of chylous effusion.

Management of chylous ascites depends on its etiology. Once UVC complications are suspected, prompt removal of the catheter is required ${ }^{1}$. Other measures may include conservative care or active treatments, including administration of anticoagulants and thrombolytics to prevent further life-threatening complications, which mainly occur in the case of higher positioned UVCs within heart chambers and lung vessels ${ }^{1}$. To reduce and prevent this potential risk of severe UVC complications, prolonged UVC use should be avoided. Repeated ultrasonography examinations in addition to plain radiography should be performed to confirm the UVC tip location ${ }^{1,10,11)}$. Abiramalatha et al. ${ }^{1)}$ emphasized the significance of repeated and regular bedside echocardiographic screening (once every 2 or 3 days while the UVC is in place) to monitor the location of the UVC tip, which can travel over time, especially for early detection of catheter-related cardiac complications.

Consequently, our case suggests that TPN extravasation from UVC should be considered when chyloperitoneum occurs in newborns with an indwelling catheter. Regular monitoring of UVC positions is crucial, and the UVC should be promptly removed in case the catheter is found to be malpositioned.

\section{CONFLICT OF INTEREST}

No potential conflict of interest relevant to this article was reported.

\section{REFERENCES}

1. Abiramalatha T, Kumar M, Shabeer MP, Thomas N. Advantages of being diligent: lessons learnt from umbilical venous catheterisation in neonates. BMJ Case Rep 2016;2016:bcr2015214073.

2. Mutlu M, Aslan Y, Kul S, Yilmaz G. Umbilical venous catheter complications in newborns: a 6-year single-center experience. J
Matern Fetal Neonatal Med 2016;29:2817-22.

3. Abiramalatha T, Kumar M, Shabeer MP. Pleural effusion caused by a malpositioned umbilical venous catheter in a neonate. BMJ Case Rep 2015;2015:bcr2015212705.

4. Wu J, Mu D. Vascular catheter-related complications in newborns. J Paediatr Child Health 2012;48:E91-5.

5. Hermansen MC, Hermansen MG. Intravascular catheter complications in the neonatal intensive care unit. Clin Perinatol 2005;32:141-56.

6. Tarn AC, Lapworth R. Biochemical analysis of ascitic (peritoneal) fluid: what should we measure? Ann Clin Biochem 2010;47(Pt5):397-407.

7. Karagol BS, Zenciroglu A, Gokce S, KundakAA, Ipek MS. Therapeutic management of neonatal chylous ascites: report of a case and review of the literature. Acta Paediatr 2010;99:1307-10.

8. Lopez-Gutierrez JC, Tovar JA. Chylothorax and chylous ascites: management and pitfalls. Semin Pediatr Surg 2014;23:298-302.

9. Shukla H, Ferrara A. Rapid estimation of insertional length of umbilical catheters in newborns. Am J Dis Child 1986;140:7868.

10. Michel F, Brevaut-Malaty V, Pasquali R, Thomachot L, Vialet R, Hassid S, et al. Comparison of ultrasound and X-ray in determining the position of umbilical venous catheters. Resuscitation 2012;83:705-9.

11. Ades A, Sable C, Cummings S, Cross R, Markle B, Martin G. Echocardiographic evaluation of umbilical venous catheter placement. J Perinatol 2003;23:24-8.

12. Dunn PM. Localization of the umbilical catheter by post-mortem measurement. Arch Dis Child 1966;41:69-75.

13. Tsui BC, Richards GJ, Van Aerde J. Umbilical vein catheterization under electrocardiogram guidance. Paediatr Anaesth 2005;15: 297-300.

14. Hagerott HE, Kulkarni S, Restrepo R, Reeves-Garcia J. Clinicalradiologic features and treatment of hepatic lesions caused by inadvertent infusion of parenteral nutrition in liver parenchyma due to malposition of umbilical vein catheters. Pediatr Radiol 2014;44:810-5.

15. Gulcan H, Hanta D, Torer B, Temiz A, Demir S. Hepatic laceration as a life-threatening complication of umbilical venous catheterization. Turk J Pediatr 2011;53:342-5.

16. Guzoglu N, Erdeve O, Yilmaz Y, Dilmen U. Intraperitoneal extravasation from umbilical venous catheter in differential diagnosis of neonatal chylous ascites. Acta Paediatr 2010;99: 1284.

17. Shareena I, Khu YS, Cheah FC. Intraperitoneal extravasation of total parental nutrition infusate from an umbilical venous catheter. Singapore Med J 2008;49:e35-6.

18. Coley BD, Seguin J, Cordero L, Hogan MJ, Rosenberg E, Reber K. Neonatal total parenteral nutrition ascites from liver erosion by 
umbilical vein catheters. Pediatr Radiol 1998;28:923-7.

19. Ramasethu J. Complications of vascular catheters in the neonatal intensive care unit. Clin Perinatol 2008;35:199-222.

20. Cohen M, Sprigg A, Roberts I, Bustani P. Subcapsular haema- toma and multifocal necrosis as fatal liver complications following umbilical vein catheterisation in a premature baby. Eur J Pediatr Surg 2006;16:55-7. 